\title{
Prenatal Ultrasonography and Early Surgery for Congenital Cystic Disease of the Lung
}

\author{
By C.A. dell'Agnola, B. Tadini, F. Mosca, and J.R. Wesley \\ Milan, Italy and Ann Arbor, Michigan
}

\begin{abstract}
- With the recent advent of prenatal ultrasound as a routine screening procedure, diagnosis of congenital cystic lung disease has been made in utero, raising the possibility of elective surgery for these lesions early in infancy before the patient develops respiratory distress or potentially lifethreatening infection. From 1979 to 1989 six cases of congenital lung cyst were diagnosed in utero by prenatal ultrasound and followed during pregnancy. Two of the six were not confirmed after birth because the mothers preferred an abortion. The remaining four cases were studied periodically during gestation by ultrasonography. At birth, the first infant developed respiratory distress and underwent urgent left upper lobectomy and left lower segmentectomy at age 18 hours. The other three underwent elective lobectomy at age 10 days, 3 months, and 7 months, respectively. The fourth infant had a normal chest $x$-ray and ultrasound at birth, and the congenital cysts were confirmed by computed tomography scan. The pathological diagnosis in all four cases was cystic adenomatoid malformation. In two cases, intraoperative measurement of pulmonary function demonstrated significant improvement after resection of the affected lobe. We conclude that congenital lung cysts can be accurately diagnosed by prenatal ultrasound "screening" as early as 18 to 24 weeks' gestation. Advantages of early diagnosis include the option of moving the mother and unborn child to a high-risk obstetrical center for urgent operation on the newborn infant if necessary. Otherwise, once the diagnosis is confirmed, surgical correction can be performed electively and safely before respiratory distress or pulmonary infection complicates the infant's growth and development.

Copyright $\odot 1992$ by W.B. Saunders Company
\end{abstract}

INDEX WORDS: Congenital cystic lung disease, prenatal ultrasound.

C ONGENITAL cystic disease of the lung includes four classic lesions that may present as abnormal cystic areas within the pleural cavity in early life: congenital adenomatoid malformation (CAM), pulmonary sequestration, congenital lobar overinflation, and bronchogenic cyst. Although histologically distinct, they share similar clinical and embryological characteristics, are often difficult to diagnose, and always require surgical therapy. Diagnosis may be

From the Departments of Pediatric Surgery, and Neonatology, University of Milan, Milan, Italy, and the Section of Pediatric Surgery, Mott Children's Hospital, University of Michigan, Ann Arbor, MI.

Date accepted: June 4, 1991.

Supported in part by A.S.M. Associazione per lo Studio delle Malformazioni, Milan, Italy.

Address reprint requests to John R. Wesley, MD, Mayo Clinic, 200

First St SW, Rochester, MN 55905.

Copyright $\odot 1992$ by W.B. Saunders Company

0022-3468/92/2711-0011\$03.0010 during the newborn period, in cases in which the space-occupying cystic lesion causes respiratory distress, or delayed by months to years until the cyst becomes infected, presenting as recurrent pneumonia or lung abscess. In the past, late diagnosis and treatment was accompanied by significant morbidity and mortality. During the last 10 years, pediatric surgeons have found that early diagnosis and prompt surgical treatment are both safe and effective. ${ }^{1}$

With the recent advent of prenatal ultrasound as a routine screening procedure, diagnosis of congenital cystic lung disease has been made in utero, raising the possibility of elective surgery for these lesions early in infancy before the patient develops respiratory distress or potentially life-threatening infection. ${ }^{2}$ The present study reviews four infants diagnosed with congenital cystic lung disease by prenatal ultrasound with three operated on electively before becoming symptomatic.

\section{MATERIALS AND METHODS}

From 1979 to 1989 , six cases of congenital lung cyst were diagnosed in utero by prenatal ultrasound and followed during pregnancy in the Department of Pediatric Surgery, University of Milan. ${ }^{2}$ Two of the six were not confirmed by us after birth because the mothers preferred an abortion. The remaining four cases were studied periodically during gestation and operated on electively after birth.

Segmentectomy or lobectomy was carried out at operation, and in all four cases a type I CAM was diagnosed. ${ }^{3}$

In two of the four cases (see cases 2 and 4), respiratory function was measured immediately before and after the operation.

A Servoventilator $900 \mathrm{C}$ (Siemens) and a computerized FLEISCH pneumotocograph Type 00 (PN 0079)-Pulmonary evaluation and diagnostic system (PEDS) with two pressure-transducers (for endotracheal and endoesophageal pressure, respectively) were used to study the pulmonary function in cases 2 and 4 just before (time $0=\mathrm{T} 0$ ) and 30 minutes after the operation under anesthesia (time $30=\mathrm{T} 30$ ).

The following parameters were evaluated: respiratory frequency, tidal volume, pulmonary compliance, transpulmonary pressure, total resistance, and total work. Upon integrating these parameters, pressure-volume loops were obtained in both cases.

The prenatal evolution of the lung cyst was followed periodically in our department by means of real-time ultrasonography with cine tape recording until the time of delivery.

Three of four women delivered vaginally at term. The fourth underwent cesarean section, not because of fetal pathology, but electively because of a previous delivery by cesarean section.

\section{Case Reports}

Case 1. The prenatal diagnosis of congenital lung cyst was made at the 2/th week of gestation by routine fetal ultrasound (Fig 

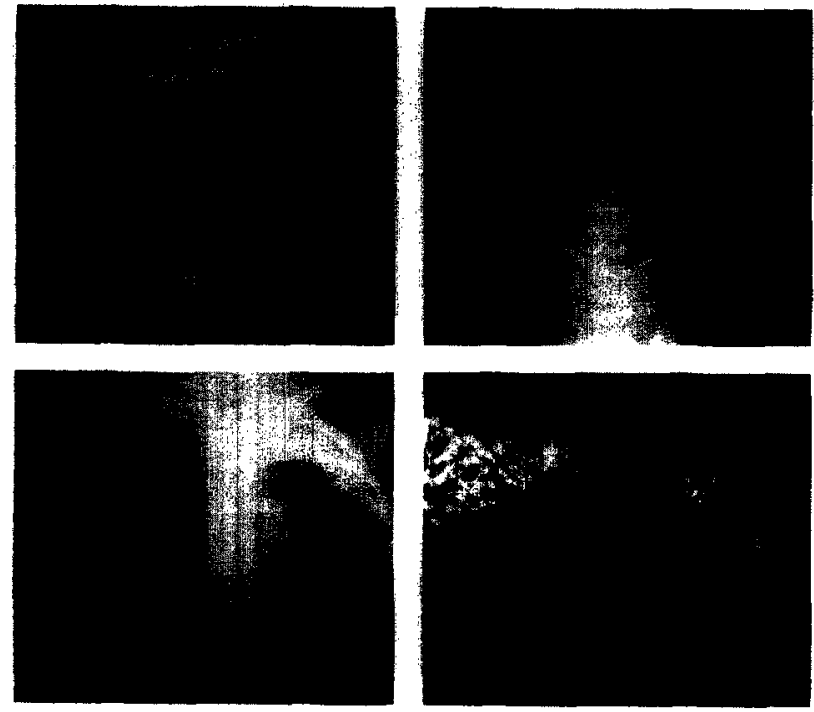

Fig 1. (A) Prenatal ultrasonography and (B) preoperative $x$-ray in case 1. (C) Plain $x$-ray 4 years after operation. (D) Operative specimen showing multiple cysts.

1). The large left-sided cyst displaced the heart and mediastinum, but neither hydrothorax nor anasarca occurred during pregnancy. Cesarean section was performed at the 40th week with delivery of a 2.6-kg female infant. Progressive respiratory distress developed, and a plain chest $x$-ray confirmed the prenatal diagnosis of congenital lung disease. At age 18 hours, a left upper lobectomy and a double segmentectomy (apical and antero medial segments) of the left lower lobe was performed with removal of multiple large cysts. Respiratory function dramatically improved, and the baby was extubated 24 hours after surgery. CAM was diagnosed by pathological examination. Follow-up over 4 years showed an active, well-developed girl with apparent normal pulmonary function.

Case 2. The prenatal diagnosis of congenital lung cyst was made by routine fetal ultrasound at the 20th week of gestation (Fig 2). A large cyst involved the left lower lung. Neither hydrothorax nor anasarca were observed during the remaining gestation. The
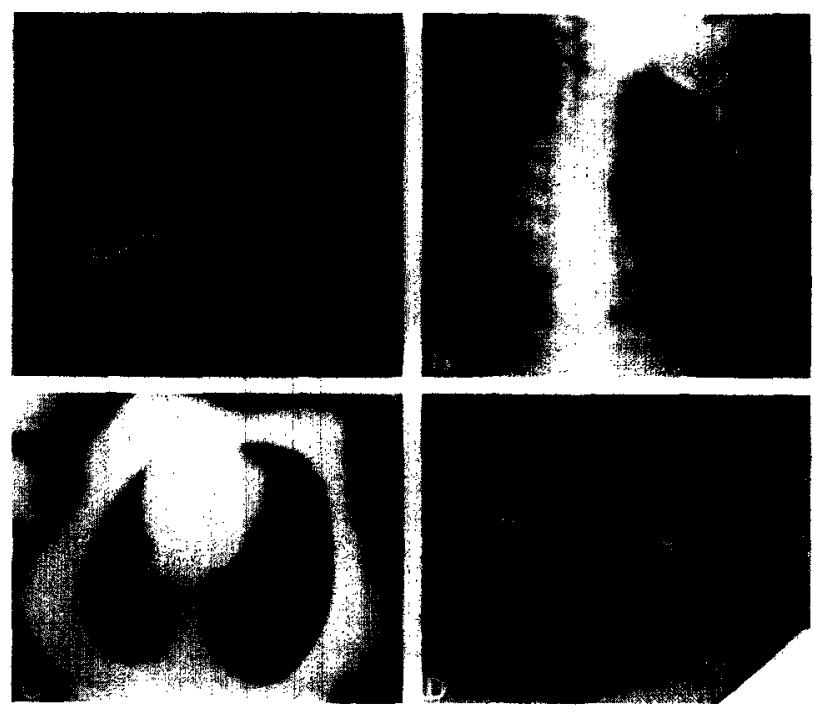

Fig 2. (A) Prenatal ultrasonography and (B) preoperative plain $x$-ray in case 2. (C) $C T$ scan confirmed the CAM. (D) Operative specimen, showing large cyst (arrow). pregnancy was otherwise uneventful, and spontaneous vaginal delivery of a $3.2-\mathrm{kg}$ girl occurred at the 39 th week. The female infant was free of respiratory symptoms. Plain $\mathrm{x}$-ray and computed tomography (CT) scan confirmed the prenatal diagnosis suggesting CAM. A left lower lobectomy was successfully performed on the 10th day of life. Pulmonary function measured at the time of surgery improved immediately and significantly after lobectomy, and the postoperative course was uneventful. Pathological examination confirmed CAM. Follow-up at 11 months showed a healthy infant with apparent normal pulmonary function.

Case 3. The prenatal diagnosis of congenital lower lung cyst was made at the 18th week of gestation and followed by periodic ultrasonography until birth (Fig 3). The differential diagnosis initially included pulmonary sequestration, but the typical image of cysts at the 19th week strongly supported the diagnosis of CAM.2 The cysts did not increase in size during gestation, and no fetal complications were observed. Term vaginal delivery produced a $3.9-\mathrm{kg}$ boy with no evidence of respiratory distress. X-ray at birth was equivocal for right lower lung cysts, but these were demonstrated later by uitrasound and $C T$ scan. The child remained free of symptoms and underwent surgery at 3 months of age. At surgery the right lower lobe was distorted by one large cyst and two smaller cysts with the clinical appearance of CAM. A right lower lobectomy was performed. Due to technical difficulties with measurement, the improvement of the pulmonary compliance, although appreciated clinically, could not be reliably recorded. Histological examination confirmed the diagnosis of CAM. The postoperative course was uneventful, and the infant was doing very well with no respiratory symptoms at the 3-month follow-up visit.

Case 4. The prenatal diagnosis of congenital lung cyst was made at the 20th week of gestation (Fig 4). Follow-up ultrasonography showed nomal fetal growth without hydrothorax or anasarca. At term, a 3.4-kg girl was born by spontaneous vaginal delivery and had no respiratory symptoms. The cysts could not be demonstrated after birth either by plain chest $x$-ray or by ultrasonography. However, $\mathrm{CT}$ scan confirmed the prenatal diagnosis, and the infant underwent surgery at age 7 months. A left lower lobectomy was performed, and intraoperative measurement of pulmonary func-
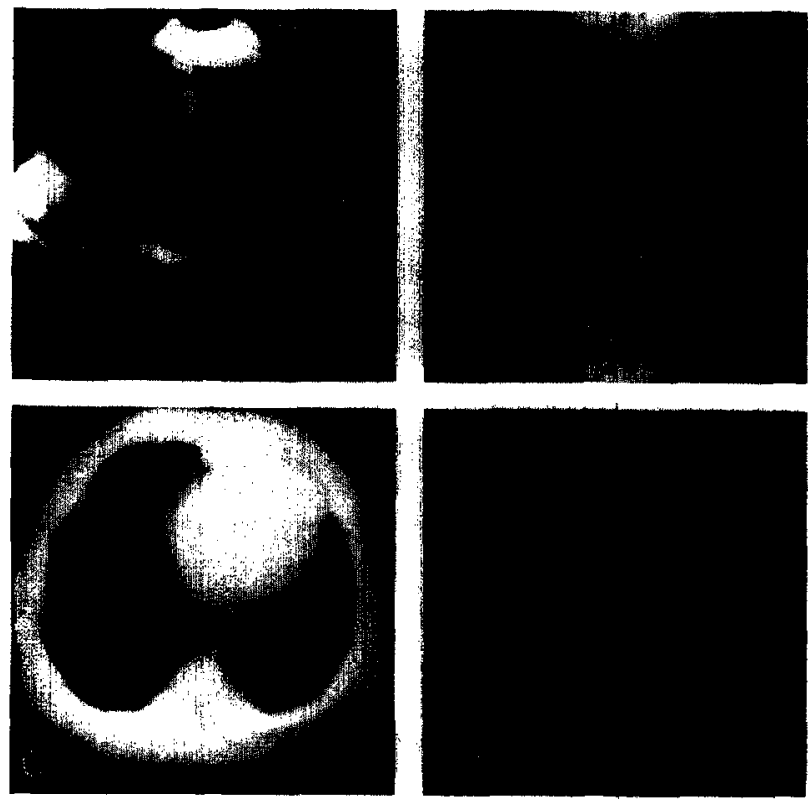

Fig 3. (A) Prenatal uitrasonography and (B) equivocal postnatal $x$-ray in case 3 . (C) The CT scan shows the cysts. (D) Operative specimen. 

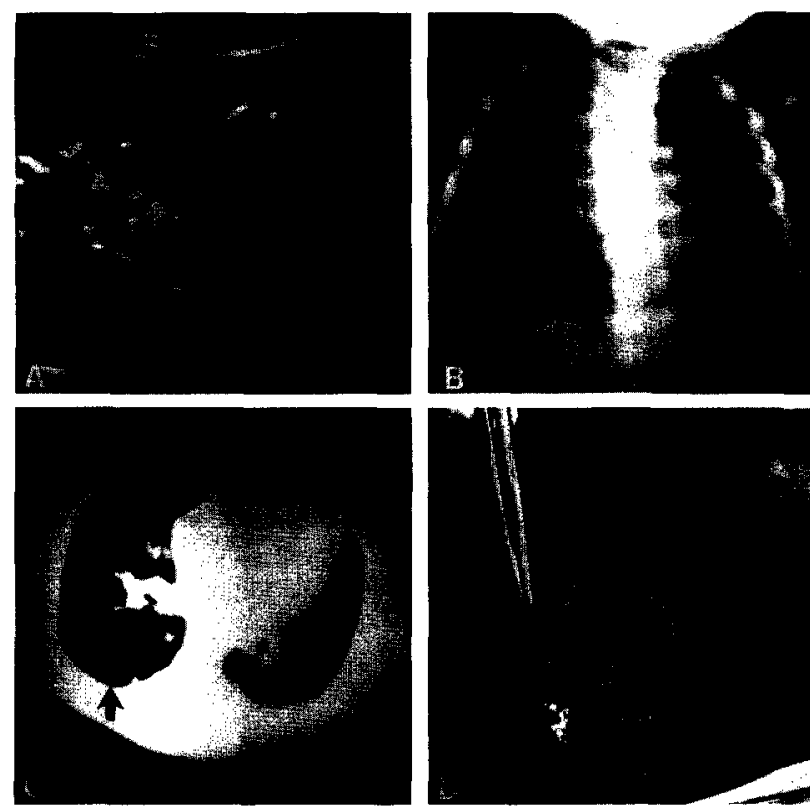

Fig 4. (A) Prenatal ultrasonography in case 4. (B) The x-ray after birth doesn't show the cysts prenatally observed at US. (C) CT scan demonstrates the lung cysts. (D) Surgical specimen (forceps point to CAM).

tion demonstrated significant improvement after resection of the affected lobe. The postoperative course was uneventful, and histological examination confirmed the diagnosis of CAM. Follow-up 3 months from the time of operation was entirely normal.

\section{RESULTS}

The prenatal diagnosis of congenital cystic lung disease was confirmed at birth, and the diagnostic impression of CAM was confirmed by histological examination at time of surgery in all four cases. In each of the four infants, the cysts did not increase in size during fetal life. In two cases we observed the evolution of the cystic abnormality from the 18 th and 20th week onward, respectively. Although no further in utero complications were observed in patient 1 , she was shown to have multiple large cysts displacing the mediastinum at 24 weeks, and progressive respiratory distress during the first 18 hours of life led to urgent surgery. In the 24 hours following operation, there was dramatic clinical improvement in respiratory function, as previously described. In cases 2 and 4, intraoperative measurement of pulmonary function showed less dramatic but significant improvement immediately after removing the affected lobes. In both cases, a significant improvement in both tidal volume and compliance after lobectomy at T30 was seen (Table 1). This is shown by the "sigmoid shape" slope in the inspiratory half of the curve, which reflects a larger volume at the same pressure after the operation (Fig 5). Because of technical measurement
Table 1. Preoperative and Postoperative Data from Cases 2 and 4

\begin{tabular}{lccccc}
\hline & \multicolumn{2}{c}{ Case 2 } & & \multicolumn{2}{c}{ Case 4 } \\
\cline { 2 - 3 } \cline { 5 - 6 } & $\begin{array}{c}\text { Pre- } \\
\text { operative }\end{array}$ & $\begin{array}{c}\text { Post- } \\
\text { operative }\end{array}$ & $\begin{array}{c}\text { Pre- } \\
\text { operative }\end{array}$ & $\begin{array}{c}\text { Post- } \\
\text { operative }\end{array}$ \\
\hline $\mathrm{T}(\mathrm{min})$ & 0 & 30 & & 0 & 30 \\
$\mathrm{Rf}(\mathrm{breaths} / \mathrm{min})$ & 30 & 30 & & 31.4 & 31.4 \\
$\mathrm{Vt}(\mathrm{mL} / \mathrm{kg})$ & 5.1 & 8 & & 4.6 & 5.8 \\
$\mathrm{Ct}\left(\mathrm{mL} / \mathrm{cm} \mathrm{H}_{2} \mathrm{O} / \mathrm{kg}\right)$ & 0.248 & 0.422 & & 0.312 & 0.362 \\
$\mathrm{P}\left(\mathrm{cm} \mathrm{H} \mathrm{H}_{2} \mathrm{O}\right)$ & 22 & 22 & & 16.5 & 16.5 \\
$\mathrm{Rt}\left(\mathrm{cm} \mathrm{H} \mathrm{H}_{2} \mathrm{O} / \mathrm{L} / \mathrm{s}\right)$ & 152 & 59.1 & & 56.1 & 63 \\
$\mathrm{Wt}(\mathrm{g} \mathrm{cm} / \mathrm{kg})$ & 216 & 137 & & 183 & 137.2 \\
\hline
\end{tabular}

Abbreviations: Rf, respiratory frequency; $\mathrm{Vt}$, tidal volume; $\mathrm{Ct}$, compliance; $P$, pressure; $R t$, transpulmonary resistance; $W t$, total work.

difficulties during the third case, this demonstration was not possible.

Lobectomy in thrce cases and combined lobectomysegmentectomy in one case were more readily accomplished because of the excellent condition of the lung parenchyma adjacent to the cysts; namely, neither atelectasis nor inflammatory adhesions of the nearby segments or lobes were observed.

Surgery was easily performed without significant blood loss. In all cases, the remaining lung tissue reexpanded to fill the hemithorax within a few hours
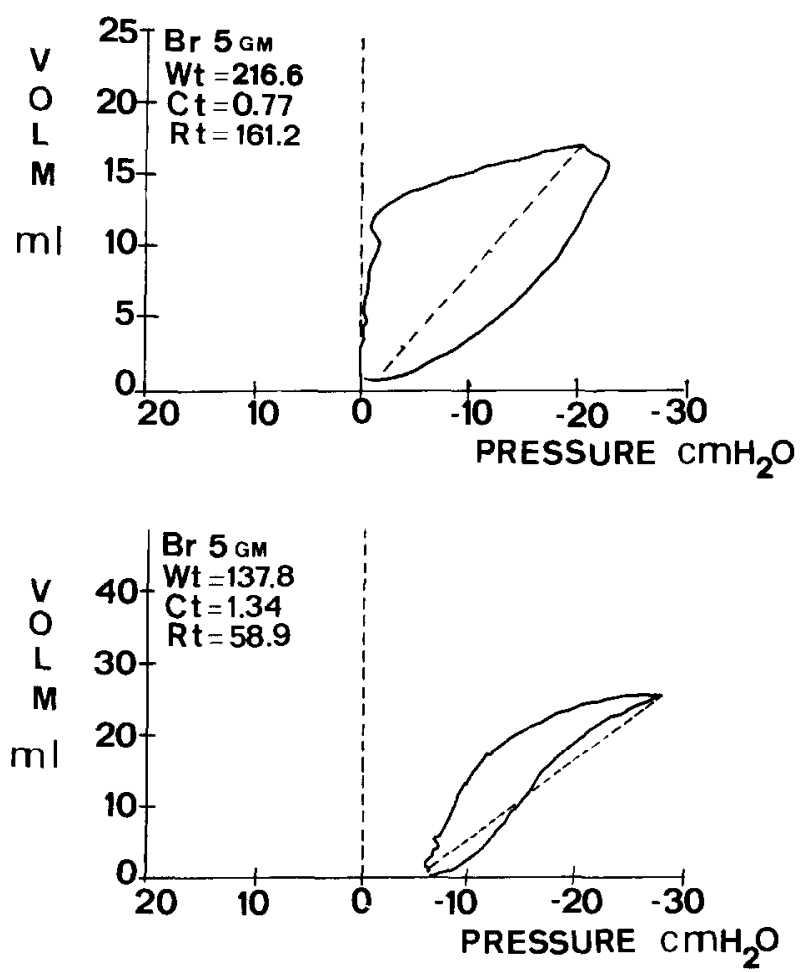

Fig 5. Volume-pressure loops in case 2. Preoperative (top) and postoperative findings (bottom) at the 5 th breath ( $\mathrm{Br} 5 \mathrm{th}$ ). Remarkable improvement of tidal volume $(\mathrm{Vt})$ and compliance $(\mathrm{Ct})$ after lobectomy. This is shown by the "sigmoid shape" slope in the inspiratory branch which reflects a larger volume at the same pressure after the operation. [001a] 
of the operation. A silastic chest tube was placed at the time of surgery in each case and removed 5 days after the operation.

Follow-up of these four patients at 4 years, 11 months, 3 months, and 3 months, respectively, shows apparent normal function of the remaining pulmonary tissue. All children are growing and free of respiratory infection.

\section{DISCUSSION}

With the advancing technology and ease of prenatal ultrasound, congenital cysts of the lung can be diagnosed with increasing accuracy between 18 and 24 weeks of gestational age. The advantages of prenatal diagnosis of these lesions include the option of moving the mother and unborn child to a high-risk obstetrical center where delivery can be planned with expert neonatologists, pediatric anesthesiologists, and pediatric surgeons available. Once the diagnosis is confirmed, surgical correction can be carried out electively before respiratory distress or pulmonary infection complicate the infant's growth and development. ${ }^{12,4,5}$ Postnatal plain chest $\mathrm{x}$-ray is not always sufficient to diagnose congenital lung cyst, nor is postnatal ultrasound always sufficient, as we discovered in case 4. Rather, CT scan was required to demonstrate the congenital lung cysts in our fourth patient, an important point in confirming the accuracy of carefully performed prenatal ultrasonography.

We followed all fetuses from the time of prenatal ultrasound diagnosis of congenital lung cysts to the birth of the infant. Although the four diagnoses were made early in pregnancy, the cysts did not increase during fetal life and neither hydrothorax nor anasarca occurred.
If the evolution of the congenital cyst remains uncomplicated during fetal life, the infant can be delivered at term and elective surgery subsequently carried out. Since all congenital lung cysts eventually require surgery, a precise diagnosis as to specific type (CAM, pulmonary sequestration, congenital lobar overinflation, or bronchogenic cyst) is not important, even though we correctly predicted the diagnosis of CAM in our four cases, as confirmed by pathological examination.

Early elective operation is preferred before respiratory distress or infection complicate the procedure. Surgery carried out in our four cases was straightforward and unencumbered by adhesions or destroyed tissue planes which characteristically affect patients diagnosed after multiple bouts of pulmonary infections. ${ }^{1,6,7}$ Moreover, in two of the three asymptomatic cases, pulmonary function improved immediately after lobectomy, as documented intraoperatively prior to thoracotomy and 30 minutes postoperatively under anesthesia.

We conclude that the advantages of early surgery for congenital cystic lung disease are manifest by our experience with the four cases reported. Prenatal ultrasound is an accurate and important screening procedure; and, once a prenatal diagnosis of cystic lung disease has been established, CT scan should be obtained to confirm the diagnosis whenever a chest $\mathrm{x}$-ray or repcat ultrasound is equivocal or "normal" after birth. Definite improvement in pulmonary function can be expected (as measured in cases 2 and 4) after early elective surgery for congenital lung cyst, and the otherwise inevitable complications of respiratory distress or recurrent infection can be avoided.

\section{REFERENCES}

1. Wesley IR, Heidelherg KP, DiPietro MA, et al: Diagnosis and management of congenital cystic disease of the lung in children. J Pediatr Surg 21:202-207, 1986

2. Deffrenne P, Coicaud C, Pracros JP, et al: Malformation adenomatoide pulmonaire. Diagn Prenatal Chir Pediatr 26:287293, 1985

3. Stocker JT, Madewell JE, Drake RM: Congenital cystic adenomatoid malformation of the lung. Hum Pathol 8:155-171. 1977

4. Monclaire T, Schistad G: Congenital pulmonary cysts versus a differential diagnosis in the newborn: Diaphragmatic hernia. J Pediatr Surg 9:417-418, 1974

5. Muayed R, Azmy AF, Fyfe AMB, et al: Congenital cystic adenomatoid malformation of the lung. Potential diagnostic pitfall. Z Kinderchir 41:107-108, 1986

6. Lilly JR, Wesenberg RL, Shikes RM: Segmental lung resection in the first year of life. Ann Thorac Surg 22:16-22, 1976

7. Buntain WL, Isaacs H, Payne VC, et al: Lobar emphysema, cystic adenomatoid malformation, pulmonary sequestration, and bronchogenic cyst in infancy and children: A clinical group. J Pediatr Surg 9:85-93, 1974 